Louisiana State University

LSU Digital Commons

Faculty Publications

Department of Physics \& Astronomy

7-10-2012

\title{
Optical and infrared analysis of type II SN 2006bc
}

Joseph S. Gallagher

University of Cincinnati

B. E.K. Sugerman

Goucher College

Geoffrey C. Clayton

Louisiana State University

J. E. Andrews

Louisiana State University

J. Clem

Louisiana State University

See next page for additional authors

Follow this and additional works at: https://digitalcommons.Isu.edu/physics_astronomy_pubs

\section{Recommended Citation}

Gallagher, J., Sugerman, B., Clayton, G., Andrews, J., Clem, J., Barlow, M., Ercolano, B., Fabbri, J., Otsuka, M., Wesson, R., \& Meixner, M. (2012). Optical and infrared analysis of type II SN 2006bc. Astrophysical Journal, 753 (2) https://doi.org/10.1088/0004-637X/753/2/109

This Article is brought to you for free and open access by the Department of Physics \& Astronomy at LSU Digital Commons. It has been accepted for inclusion in Faculty Publications by an authorized administrator of LSU Digital Commons. For more information, please contact ir@lsu.edu. 


\section{Authors}

Joseph S. Gallagher, B. E.K. Sugerman, Geoffrey C. Clayton, J. E. Andrews, J. Clem, M. J. Barlow, B. Ercolano, J. Fabbri, M. Otsuka, R. Wesson, and M. Meixner 


\title{
OPTICAL AND INFRARED ANALYSIS OF TYPE II SN 2006bc
}

\author{
Joseph S. Gallagher ${ }^{1}$, B. E. K. Sugerman ${ }^{2}$, Geoffrey C. Clayton ${ }^{3}$, J. E. Andrews ${ }^{3}$, J. Clem ${ }^{3}$, M. J. Barlow ${ }^{4}$,

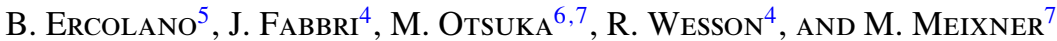 \\ ${ }^{1}$ MPCS Department, University of Cincinnati, Blue Ash College, 9555 Plainfield Road, Blue Ash, OH 45236, USA; gallagj1@uc.edu \\ ${ }^{2}$ Department of Physics and Astronomy, Goucher College, 1021 Dulaney Valley Road, Baltimore, MD 21204, USA; ben.sugerman@ goucher.edu \\ ${ }^{3}$ Department of Physics and Astronomy, Louisiana State University, 202 Nicholson Hall, Baton Rouge, LA 70803, USA; \\ gclayton@fenway.phys.lsu.edu, jandrews@phys.lsu.edu, jclem@phys.lsu.edu \\ ${ }^{4}$ Department of Physics and Astronomy, University College London, Gower Street, London WC1E 6BT, UK; \\ mjb@star.ucl.ac.uk, jfabbri@star.ucl.ac.uk, rwesson@star.ucl.ac.uk \\ ${ }^{5}$ Universitäts-Sternwarte München, Scheinerstrasse 1,81679 München, Germany; ercolano@usm.uni-muenchen.de \\ ${ }^{6}$ Institute of Astronomy and Astrophysics, Academia Sinica, P.O. Box 23-141, Taipei 10617, Taiwan, Republic of China; otsuka@ asiaa.sinica.edu.tw \\ ${ }^{7}$ Space Telescope Science Institute, 3700 San Martin Drive, Baltimore, MD 21218, USA; otsuka@stsci.edu, meixner@stsci.edu \\ Received 2011 August 5; accepted 2012 May 4; published 2012 June 19
}

\begin{abstract}
We present nebular phase optical imaging and spectroscopy and near/mid-IR imaging of the Type II SN $2006 \mathrm{bc}$. Observations reveal the central wavelength of the symmetric $\mathrm{H} \alpha$ line profile to be redshifted with respect to the host galaxy $\mathrm{H} \alpha$ emission by day 325. Such a phenomenon has been argued to result from an asymmetric explosion in the iron-peak elements resulting in a larger mass of ${ }^{56} \mathrm{Ni}$ and higher excitation of hydrogen on the far side of the supernova $(\mathrm{SN})$ explosion. We also observe a gradual blueshifting of this $\mathrm{H} \alpha$ peak which is indicative of dust formation in the ejecta. Although showing a normal peak brightness, $V \sim-17.2$, for a core-collapse SN, 2006bc fades by $\sim 6$ mag during the first 400 days suggesting either a relatively low ${ }^{56} \mathrm{Ni}$ yield, an increase in extinction due to new dust, or both. A short-duration flattening of the light curve is observed from day 416 to day 541 suggesting an optical light echo. Based on the narrow time window of this echo, we discuss implications on the location and geometry of the reflecting interstellar medium. With our radiative transfer models, we find an upper limit of $2 \times$ $10^{-3} M_{\odot}$ of dust around SN 2006bc. In the event that all of this dust were formed during the SN explosion, this quantity of dust is still several orders of magnitude lower than that needed to explain the large quantities of dust observed in the early universe.
\end{abstract}

Key words: circumstellar matter - dust, extinction - supernovae: individual (SN 2006bc)

\section{INTRODUCTION}

The majority of dust formed in galaxies at the current epoch originates in the stellar winds of low-mass, asymptotic giant branch (AGB) stars (Gehrz 1989). Although the effective temperature of AGB stars, around $3000 \mathrm{~K}$, exceeds the condensation temperature for most compositions of dust grains, pulsation or convection-driven shock waves propagating radially from the star can create transitory dense, cool layers that are suitable for dust formation. Once created the newly formed dust can be ushered away into the interstellar medium (ISM) via radiation pressure.

Recent submillimeter observations of high-redshift quasistellar objects and galaxies have revealed large quantities of dust at $z>5$ (Bertoldi et al. 2003; Dwek et al. 2007). Such redshifts correspond to an age of the universe of $1 \mathrm{Gyr}$ or less, considerably younger than the expected delay time for dust formation in AGB stars (Michałowski et al. 2010). An alternate mechanism for the creation of this high-redshift dust is dust formation in the ejecta of supernovae ( $\mathrm{SNe}$ ) given that the delay time associated with $\mathrm{SNe}$ is measured in millions, not billions, of years. This was first suggested as a possible mechanism by Cernuschi et al. (1967). Recent theoretical models predict that $0.1-1 M_{\odot}$ of dust should form in the ejecta of every SN event (Todini \& Ferrara 2001; Nozawa et al. 2003), a production rate that can certainly account for the large amount of dust at high redshift (Morgan \& Edmunds 2003; Maiolino et al. 2006; Dwek et al. 2007). Observational evidence of SN dust formation was first seen within the ejecta of SN 1987A (Lucy et al. 1991; Meikle et al. 1993; Ercolano et al. 2007). This evidence came in the form of (1) the development of an infrared excess,
(2) an increase in the optical extinction, and (3) the appearance of blueshifted emission line profiles due to the greater attenuation of emission from the far-side, redshifted ejecta emission.

To date, evidence of dust formation has been seen in all types of core-collapse $\mathrm{SNe}$, save for type Ic, and no signs of dust formation have been observed in thermonuclear, SNe Ia. In addition to SN 1987A, dust has been shown to have condensed in the ejecta of five Type II SNe, 1990I (Elmhamdi et al. 2004), 1999em (Elmhamdi et al. 2003), 2003gd (Sugerman et al. 2006; Hendry et al. 2005; Meikle et al. 2007), 2004et (Sahu et al. 2006; Kotak et al. 2009; Fabbri et al. 2011), and 2005af (Kotak et al. 2006). In each case the quantity of dust formed was less than $10^{-3} M_{\odot}$, considerably less than that needed to explain the dust observed at high- $z$. More recently, observations of SNe exhibiting strong circumstellar interaction have revealed a secondary location, outside of the ejecta, of dust formation. These observations have shown evidence of dust formation within the cool, dense shell (CDS; Deneault et al. 2003) between the forward and reverse shocks formed by the interaction of the ejecta and circumstellar material (CSM). This mechanism has been reported in four SNe, 1998S (Pozzo et al. 2004), 2005ip (Smith et al. 2009; Fox et al. 2009), 2006jc (Smith et al. 2008; Mattila et al. 2008), 2007it (Andrews et al. 2011), and 2007od (Andrews et al. 2010). However, despite their potential to produce dust in both their ejecta and in their CDS, CSM-interacting SNe have been shown only to form comparable amounts of dust to their CSM-deficient counterparts.

Recent studies that have reevaluated the delay time limits for dust formation by AGB stars have concluded that AGB stars may be more efficient dust producers at high redshift than first expected, but they have not been able to rule out $\mathrm{SNe}$ as 


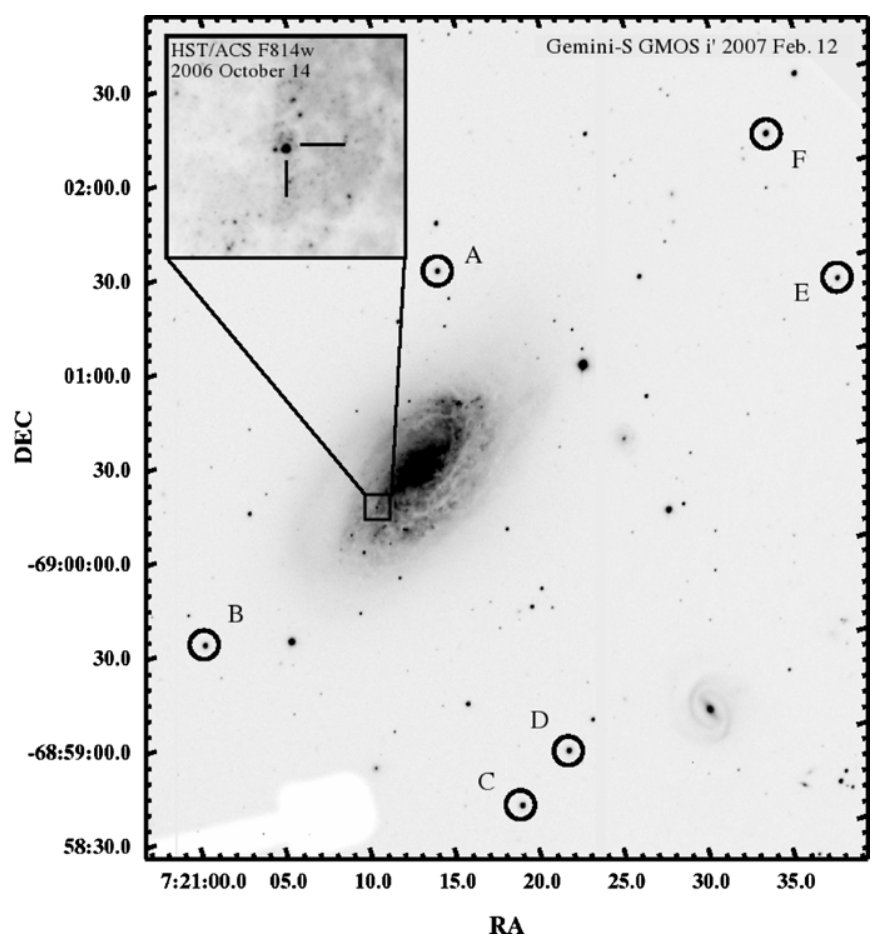

Figure 1. Gemini/GMOS-S image of the field around NGC 2397 and SN 2006bc obtained 324 days after explosion with the $i^{\prime}$ filter. A higher resolution inset showing the immediate region around SN 2006bc is shown and was obtained with HST / ACS by Smartt et al. (2009) as part of GO10498. The figure also shows the secondary standard stars that we used to transform from the instrumental to standard magnitude system (see Table 2).

major contributors (Valiante et al. 2009; Dwek \& Cherchneff 2011). Other possible, non-stellar, sources of dust have been put forward such as the growth of dust grains in the ISM. Draine (2009) suggested that although SNe contribute a portion of the dust found in the ISM, most of the dust mass observed in the ISM comes from the growth of these seed grains after they arrive in the ISM.

In spite of the great interest in understanding the origins of dust at high redshift, there is still no firm conclusion yet as to the ability of $\mathrm{SNe}$ to significantly contribute to the overall dust budget in the galaxy. The number of SNe that have been observed at multiple epochs and in multiple wavelengths is still very small, and the more SNe that are observed, the greater the realization that each $\mathrm{SN}$ is unique. This makes it of paramount importance to increase the sample of observed core-collapse $\mathrm{SNe}$, so that a statistically significant conclusion can be made on their ability to form dust. To this end, we report multitemporal, multi-wavelength observations of core-collapse SN 2006bc. In Section 2 we will describe our observing program and our reduction methods. In Section 3 we will report our results, and we summarize our conclusions in Section 4.

\section{OBSERVATIONS AND REDUCTIONS}

\subsection{Previous Observations and Relevant Statistics}

SN 2006bc was discovered on 2006 March 24.65 UT approximately $49^{\prime \prime}$ west and $43^{\prime \prime}$ north of the center of its host, NGC 2397 (Figure 1; Martin 2006). An optical spectrum taken by Patat et al. (2006) with the ESO Very Large Telescope on 2006 March 28.06 UT showed narrow Balmer emission superimposed on a blue continuum. The width of these Balmer lines suggested an expansion velocity of $\sim 2000 \mathrm{~km} \mathrm{~s}^{-1}$. Unresolved
Na I D lines originating within the Milky Way and the host galaxy were also present, and both lines had measured equivalent widths of $\sim 1.0 \AA$ (Patat et al. 2006). Patat et al. (2006) also determined a $B-V$ color for SN $2006 \mathrm{bc}$ of $\sim 0.2$ while coincident Swift Ultraviolet/Optical Telescope (UVOT) observations found $U-B \sim-0.9 \pm 0.1$ (Immler \& Brown 2006). Based on the color-SN type diagnostics of Poznanski et al. (2002), SN 2006bc is considerably bluer than most Type II SNe with colors closer to that of type Ia SNe. However, the presence of hydrogen emission and the similarities of its colors to Type II-P SN 2005cs and 2006at led to its classification as a Type II-P (Immler \& Brown 2006).

SN 2006bc was further imaged on 2006 October 14 (203 days) with the Advanced Camera for Surveys (ACS) Wide-Field Camera (WFC) in the $F 435 W, F 555 W$, and $F 814 W$ filters by Smartt et al. (2009). Alignment of these images with preexplosion images taken with the Wide Field Planetary Camera 2 (WFPC2) on board the Hubble Space Telescope (HST) on 2001 November 17 resulted in no detection of the progenitor star. However, based on the detection limit of the pre-explosion images, and assuming the progenitor to be a red supergiant, Smartt et al. (2009) derived an upper mass limit for the progenitor of SN $2006 \mathrm{bc}$ of $12 M_{\odot}$.

Based on the equivalent width measurements of the unresolved Na I D lines for the NGC 2396 and the Milky Way by (Patat et al. 2006), and using with the empirical relation of Barbon et al. (1990) relating EW(Na I D) with the intrinsic color excess, $E(B-V)$, and $R(V)=3.1$, we find a substantial total foreground extinction to SN 2006bc of $A_{v} \sim 1.6$. According to Tully et al. (2009), NGC 2397 is at a distance of $20.3 \mathrm{Mpc}$, and we calculated the recessional velocity of NGC 2397 via redshifted H II emission lines in our own optical spectra to be $(1462 \pm 12) \mathrm{km} \mathrm{s}^{-1}$. Finally, based on negative detections by Martin (2006) as late as 2006 March 16.59 UT, we adopt an explosion date that coincides with the date of discovery (JD 2453819.15).

\subsection{Optical Spectroscopy}

For this program data were taken with the Gemini South Telescope utilizing the GMOS-S instrument during the 2007A and 2007B semesters (GS-2007A-Q-4 and GS-2007B-Q-39). Dates of observation include 2007 February 12 (324 days), March 18 (358 days), May 11 (412 days), September 20/24 ( $\sim 544$ days), November 11/14 ( $\sim 596$ days), and 2008 January 16 (662 days; see Table 1). During the first three epochs, $3 \times$ $900 \mathrm{~s}$ spectra were obtained in long-slit mode. The exposure time was augmented to $3 \times 1050 \mathrm{~s}$ for the last three epochs to compensate for the expected SN optical decline. The GMOS-S spectrograph, equipped with the B600-G5323 grating, allowed for $2760 \AA$ coverage and an FWHM resolution of $\sim 3.9 \AA$. The central wavelengths of individual spectra were $5950 \AA$, $5970 \AA$, and $5990 \AA$, respectively, to allow for effective removal of chip gaps and to avoid having the gaps fall on important emission features. A 0.75 slit width was used with the B600-G5323 grating in first order along with $2 \times 2$ binning in the low gain setting.

Spectra were reduced using the $\operatorname{IRAF}^{8}$ gemini package. An example of the slit orientation from day 324 is shown in Figure 2. The inset displays a section of the two-dimensional spectrum including emission from $\mathrm{H} \alpha$ and [N II]. The figure highlights the

\footnotetext{
8 IRAF is distributed by the National Optical Astronomy Observatory, which is operated by the Association of Universities for Research in Astronomy (AURA), under cooperative agreement with the National Science Foundation.
} 
Table 1

Summary of Observations

\begin{tabular}{|c|c|c|c|c|c|}
\hline Epoch $^{\mathrm{a}}$ & JD & Telescope & Instrument & Filters & $\begin{array}{c}\text { Exp. Time } \\
\text { (s) }\end{array}$ \\
\hline $203^{b}$ & 2454023 & $H S T$ & ACS/WFC & F555W; F814W & $1500 ; 1600$ \\
\hline 324 & 2454144 & Gemini South & GMOS-S spectra & - & $3 \times 900$ \\
\hline 324 & 2454144 & Gemini South & GMOS-S imaging & $g^{\prime} r^{\prime} i^{\prime}$ & 60 \\
\hline 358 & 2454178 & Gemini South & GMOS-S spectra & - & $3 \times 900$ \\
\hline 358 & 2454178 & Gemini South & GMOS-S imaging & $g^{\prime} r^{\prime} i^{\prime}$ & 60 \\
\hline 412 & 2454232 & Gemini South & GMOS-S spectra & - & $3 \times 900$ \\
\hline 412 & 2454232 & Gemini South & GMOS-S imaging & $g^{\prime} r^{\prime} i^{\prime}$ & 60 \\
\hline 496 & 2454315 & Spitzer & IRS/PUI & Blue & 31 \\
\hline 532 & 2454353 & $H S T$ & WFPC2 & F606W; F814W & $1600 ; 1600$ \\
\hline 537 & 2454356 & Spitzer & IRAC & CH $1-4$ & 10 \\
\hline 540 & 2454359 & Spitzer & MIPS & $24 \mu \mathrm{m}$ & 31 \\
\hline 541 & 2454361 & $H S T$ & NICMOS & F110W; F160W; F205W & $640 ; 512 ; 576$ \\
\hline 544 & 2454364 & Gemini South & GMOS-S spectra & - & $3 \times 1050$ \\
\hline 544 & 2454364 & Gemini South & GMOS-S imaging & $g^{\prime} r^{\prime} i^{\prime}$ & 60 \\
\hline 596 & 2454416 & Gemini South & GMOS-S spectra & - & $3 \times 1050$ \\
\hline 596 & 2454416 & Gemini South & GMOS-S imaging & $g^{\prime} r^{\prime} i^{\prime}$ & 60 \\
\hline 662 & 2454482 & Gemini South & GMOS-S spectra & - & $3 \times 1050$ \\
\hline 662 & 2454482 & Gemini South & GMOS-S imaging & $g^{\prime} r^{\prime} i^{\prime}$ & 60 \\
\hline 648 & 2454467 & Spitzer & IRS/PUI & Blue & 31 \\
\hline 688 & 2454507 & Spitzer & MIPS & $24 \mu \mathrm{m}$ & 31 \\
\hline 690 & 2454509 & Spitzer & IRAC & CH 1-4 & 10 \\
\hline 694 & 2454514 & $H S T$ & WFPC2 & F606W; F814W & $1600 ; 1600$ \\
\hline 753 & 2454572 & Spitzer & MIPS & - & 31 \\
\hline 776 & 2454595 & Spitzer & IRAC & CH 1-4 & 27 \\
\hline
\end{tabular}

Notes.

a Assuming explosion date JD $=2453819$.

b Data taken as part of GO10498 (Smartt et al. 2009). Data were acquired from the archive, re-reduced, and analyzed.

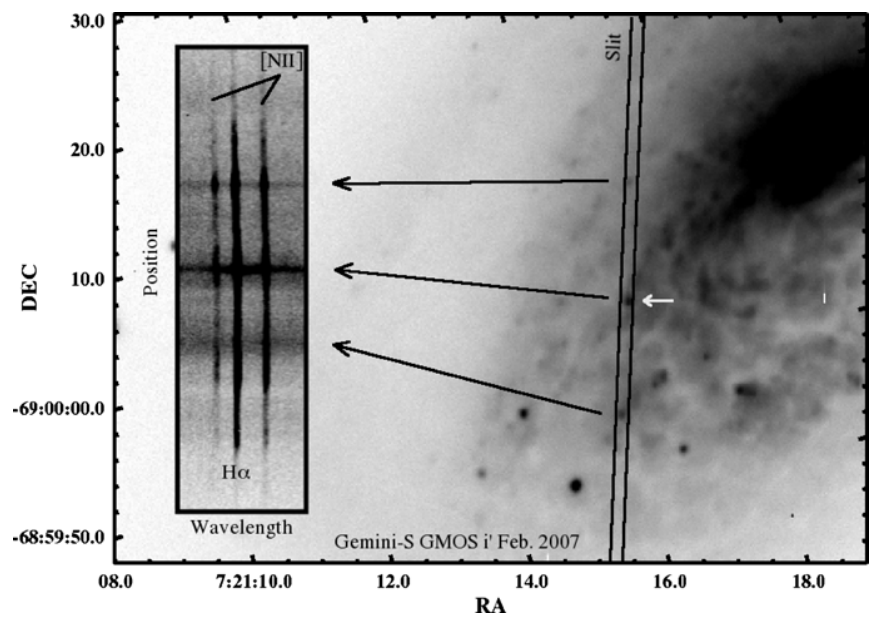

Figure 2. GMOS-S $i^{\prime}$ image of SN 2006bc from 2007 February 12. The position of the SN is marked with a white arrow. The orientation of the slit for the February spectroscopic observations is depicted. The image shows the crowded environment in which SN $2006 \mathrm{bc}$ is found, and the inset shows the section of the GMOS-S spectrum around $\mathrm{H} \alpha$. The respective origins of three emission regions along the spatial axis are marked with arrows in the image. Care was taken to avoid emission from extraneous stars or H II regions (such as those shown above and below the SN position) in the section of sky used in the background subtraction.

contamination of galactic emission in the light from the SN. The $\mathrm{SN}$ and sky regions used during the one-dimensional spectral extraction were determined by visual inspection. Care was taken when defining the regions of sky to include continuum emission from the galaxy, but to avoid stars and clumpy $\mathrm{H}$ in regions falling on the slit as shown in Figure 2. The three spectra obtained each individual night were averaged to improve signal to noise.

\subsection{Optical Imaging}

Coincident optical imaging was obtained using the GMOS-S imager in three Sloan Digital Sky Survey filters, $g^{\prime}, r^{\prime}$, and $i^{\prime}$. The images were reduced and stacked using the standard routines within the gemini package. To remove as much contamination from galactic light as possible, we performed pointspread-function (PSF)-matched difference imaging in each filter using the IRAF difimphot package (Tomaney \& Crotts 1996; Sugerman et al. 2005). The procedure entailed registering and matching the PSFs of an object and reference image. Given the lack of pre-explosion GMOS-S images of NGC 2397, we opted to use our day $662 g^{\prime} r^{\prime} i^{\prime}$ images as the reference epoch. In each filter, the reference image was subtracted from the images of the earlier epochs, resulting in a final PSF-matched difference image in $g^{\prime} r^{\prime} i^{\prime}$ for days 324, 358, 412, 544, and 596. An example is shown in Figure 3 of our day $324 i^{\prime}$ subtraction.

PSF photometry was then performed on the difference images using the IRAF daophot package. The instrumental $g^{\prime} r^{\prime} i^{\prime}$ magnitudes were converted into the standard Johnson-Cousins $V R I_{c}$ using a transformation involving a least-squares fit with a floating zero point (Welch et al. 2007). A $B V R I_{c}$ photometric sequence of secondary standards was derived for the 2006bc field. Observations of the secondary standard stars and $\sim 30$ stars from the lists of Landolt (2009) were taken with the Y4KCam CCD on the Yale $1.0 \mathrm{~m}$ telescope operating at the Cerro Tololo InterAmerican Observatory on 2008 October 28. For full details on the data reduction and the creation of our photometric sequence see Appendix A of Andrews et al. (2010). The standard stars are shown in Figure 1 and their corresponding magnitudes are reported in Table 2. PSF photometry on the non-differenced images of these secondary standard stars allowed us to derive the 

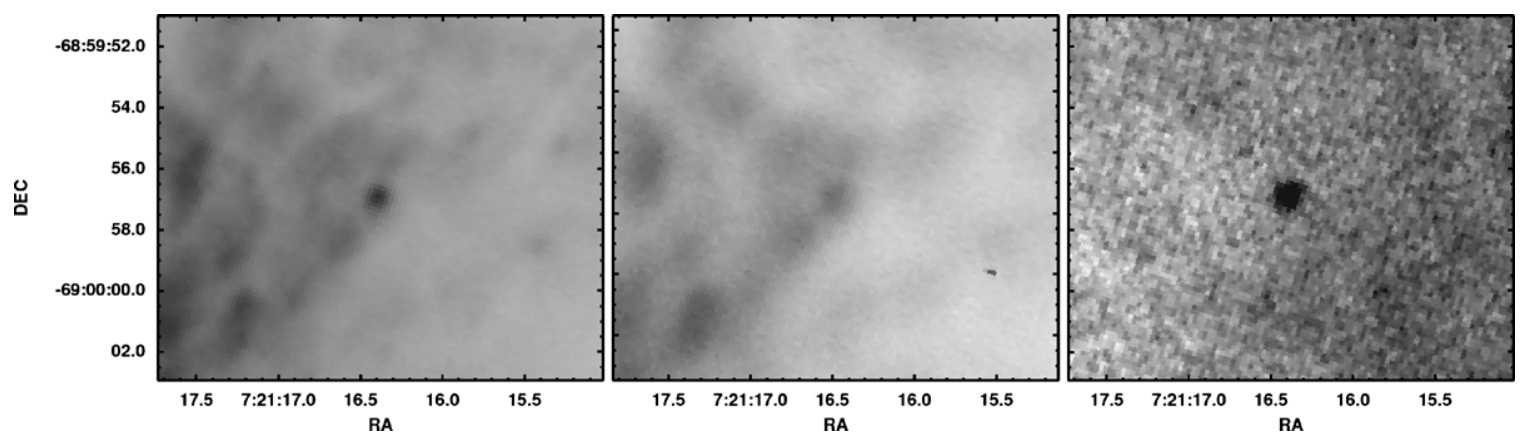

Figure 3. Example PSF-matched differenced image from our GMOS data. Left: day $324 i^{\prime}$ image of SN 2006bc. Middle: day $662 i^{\prime}$ image of SN $2006 \mathrm{bc}$. Right: final subtracted image showing the $\mathrm{SN}$ with the nebular contamination considerably reduced.

Table 2

Secondary Standard Stars

\begin{tabular}{lccc}
\hline \hline ID & $V(\delta V)$ & $R(\delta R)$ & $I(\delta I)$ \\
\hline A & $17.140(0.009)$ & $16.541(0.006)$ & $16.032(0.023)$ \\
B & $17.003(0.008)$ & $16.367(0.010)$ & $15.826(0.011)$ \\
C & $16.548(0.013)$ & $16.118(0.009)$ & $15.723(0.051)$ \\
D & $16.878(0.012)$ & $16.180(0.013)$ & $15.624(0.017)$ \\
E & $17.618(0.011)$ & $17.050(0.009)$ & $16.539(0.106)$ \\
F & $16.348(0.011)$ & $16.014(0.005)$ & $15.656(0.010)$ \\
\hline
\end{tabular}

unique, filter-dependent zero points for each night. It is important to note that any remaining SN flux present in our reference epoch will lead to our underestimating the flux at a given epoch.

Late-time imaging data were obtained by HST using WFPC2 on 2007 September 9 (532 days) and 2008 February 17 (694 days). Data were taken using the $F 606 W$ and $F 814 W$ filters, and the SN was centered on the Planetary Camera (PC) CCD chip. The images were pipeline reduced, cosmic rays were removed, and images were drizzled to a higher spatial resolution, $0^{\prime} .03$ pixel $^{-1}$ (65\% of the native pixel scale), using the STSDAS package. Instrumental magnitudes were determined via PSF-photometry using the daophot package. We generated simulated PSFs for this analysis using the PSF creation software Tiny Tim (Krist \& Hook 2004). The F606W and F814W instrumental magnitudes were transformed into Johnson $V$ and $I_{c}$, respectively, and charge transfer efficiency (CTE) corrections were applied using the iterative procedure detailed in Dolphin (2000, 2009).

\subsection{Near-infrared Imaging}

Wide band $F 110 W, F 160 W$, and $F 205 W$ images of $2006 \mathrm{bc}$ were obtained on 2007 September 17 (541 days) and 2008 February 17 (694 days) using HST equipped with the NearInfrared Camera and Multi-Object Spectrograph (NICMOS, Meixner: GO11229). The NIC2 camera employing the square wave dither pattern was utilized. The images were pipeline reduced and photometry was carried out in similar fashion to that of the WFPC2 data. Model PSFs were created with Tiny Tim and were used along with daophot to calculate instrumental magnitudes. HST system magnitudes (ST) were calculated from the measured count rate (CR) and the header keywords PHOTFLAM according to Equation (1):

$$
\mathrm{STMAG}=-2.5 \times \log _{10}(\mathrm{PHOTFLAM} \times \mathrm{CR})-21.1 .
$$

PHOTFLAM is defined as the mean flux density in units of $\operatorname{erg} \mathrm{cm}^{-2} \mathrm{~s}^{-1} \AA^{-1}$ that produces 1 count $\mathrm{s}^{-1}$ in a given $H S T$

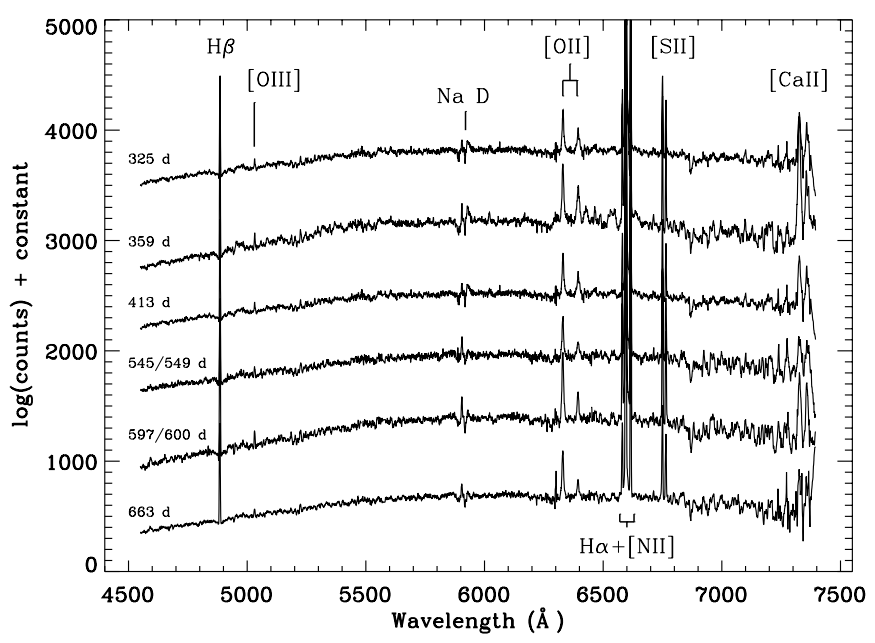

Figure 4. Gemini/GMOS-S spectra SN 2006bc spanning six epochs. Data have not been corrected for redshift or reddening.

observing mode. The ST magnitudes were then transformed into JHK magnitudes using the IRAF synphot package.

\subsection{Mid-infrared Imaging}

Three epochs of Spitzer/IRAC (3.6, 4.5, 5.8, and $8.0 \mu \mathrm{m})$ and MIPS $(24 \mu \mathrm{m})$ images were taken along with two epochs of IRS peak-up image (PUI) data at $16 \mu \mathrm{m}$. The IRAC data were taken on 2007 September 12 (537 days), 2008 February 12 (690 days), and 2008 May 8 (776 days). The IRS PUI data were taken on 2007 August 2 (496 days) and 2008 January 01 (648 days) while the MIPS data were taken on 2007 September 15 (540 days), 2008 February 10 (688 days), and 2008 April 15 (753 days). The chronology of all observations is summarized in Table 1. To improve photometric quality, all the data were mosaicked and resampled using the software package MOPEX (Makovoz $\&$ Khan 2005). The IRAC images were drizzled to 0.75 pixel $^{-1}$ ( $63 \%$ of the native pixel scale), the IRS PUI $16 \mu \mathrm{m}$ to 0.9 pixel $^{-1}$ (50\%), and the MIPS $24 \mu \mathrm{m}$ to $1^{\prime \prime} .2$ pixel $^{-1}$ (50\%). Difference imaging analysis was performed as described above with the day 776 (IRAC), day 753 (MIPS), and day 688 (IRS/PUI) images subtracted from the earlier epochs for the three instruments, respectively. PSF-photometry was performed at the SN position using DAOPHOT while a customized implementation of the IRAF package DIMPHOT, called DIPHOT (Tomaney \& Crotts 1996), was used to obtain photometric errors or upper detection limits for the differenced images. 


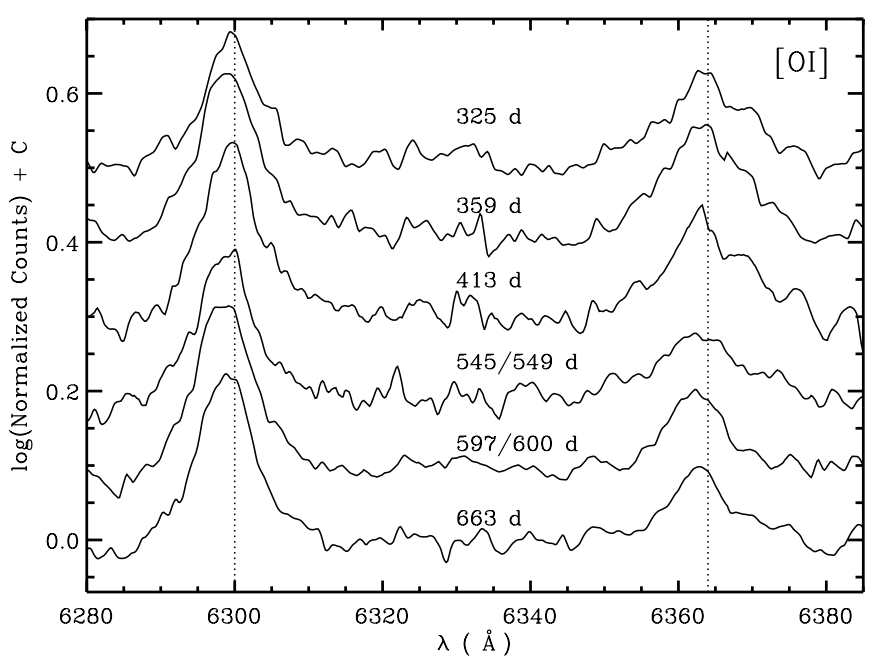

Figure 5. Evolution of the $[\mathrm{O}$ I $] \lambda 6300,6363$ emission lines across our six epochs. The spectra have been redshift corrected, but they have not been dereddened.

\section{RESULTS}

\subsection{Optical Emission Line Profiles}

As noted earlier, the spectroscopic analysis of SN 2006bc is difficult, particularly at late times, due to its location within the bright SBb host galaxy, NGC 2397. Our six spectra, stretching from days 325 to 663, are shown in Figure 4. The spectra exhibit prominent narrow emission lines of $\mathrm{H} \beta$, [O III], [N II], [S II], and $\mathrm{H} \alpha$ typically observed in the $\mathrm{H}$ II regions of star-forming galaxies. The spectra also show lines of [O I] and $\mathrm{H} \alpha$ with widths consistent with emission from the evolving $\mathrm{SN}$ ejecta (500-1000 $\mathrm{km} \mathrm{s}^{-1}$ ). The evolution of [O I] and $\mathrm{H} \alpha$ are shown in Figures 5 and 6, respectively. Although Figure 5 has been corrected for the velocity of the host galaxy, Figure 6 has not been corrected. Little change is observed in the [O I] emission profiles; however, in spite of strong contamination from nebular emission, noticeable evolution is observed in the profile of $\mathrm{H} \alpha$. Figure 6 shows the evolution of the $\mathrm{H} \alpha+[\mathrm{N}$ II] emission profile for which we have carefully aligned the narrow host galaxy $\mathrm{H} \alpha$ and $\left[\mathrm{N}_{\mathrm{II}}\right]$ lines. Apparent is the underlying emission from the SN fading over the course of our observing period.

In order to separate the nebular emission from the $\mathrm{SN}$ emission, we simultaneously fitted Gaussian curves to the blended $\mathrm{H} \alpha+[\mathrm{N}$ II $]$ profile of the host galaxy and SN. The results of this analysis are given in Figure 7. The top two panels show the fit to our day 325 profile on the left and its Gaussian components on the right. Subsequent plots show the fits to our remaining five epochs. The most obvious characteristic of the SN emission is that by day 325 the central wavelength of the SN emission is redshifted with respect to the galactic $\mathrm{H} \alpha$ emission by $\sim 800-900 \mathrm{~km} \mathrm{~s}^{-1}$. A similar phenomenon was observed in the optical spectra of SN1987A, 1999em, and 2004dj (Phillips \& Williams 1991; Elmhamdi et al. 2003; Chugai et al. 2005). In the case of SN 1987A, the emission peaks of several elements, including hydrogen, were observed to be redshifted from 600 to $1000 \mathrm{~km} \mathrm{~s}^{-1}$ during the period 150-600 days after explosion. It was subsequently argued by Chugai (1991) that this redshift was due to an asymmetry in the ${ }^{56} \mathrm{Ni}$ ejecta. Just like SN 2006bc, SN 1987A did not show this asymmetry in the [O I] emission lines, but it did show strong asymmetry in Fe II and Ni I (Meikle et al. 1989). This led Chugai (1991) to suggest that the asymmetries involved only the iron-peak elements with the redshifting of the $\mathrm{H} \alpha$ line being a secondary effect due to an increase in the excitation of hydrogen in positions where the mass of ${ }^{56} \mathrm{Ni}$ is higher. Given that we are observing the emission line profile asymmetries in $\mathrm{H} \alpha$, but not [O I], it is likely that we are seeing a similar effect in the optical spectra of SN 2006bc.

Figure 8 shows the evolution of our Gaussian model to the SN $\mathrm{H} \alpha$ emission profile with time. The inset shows the

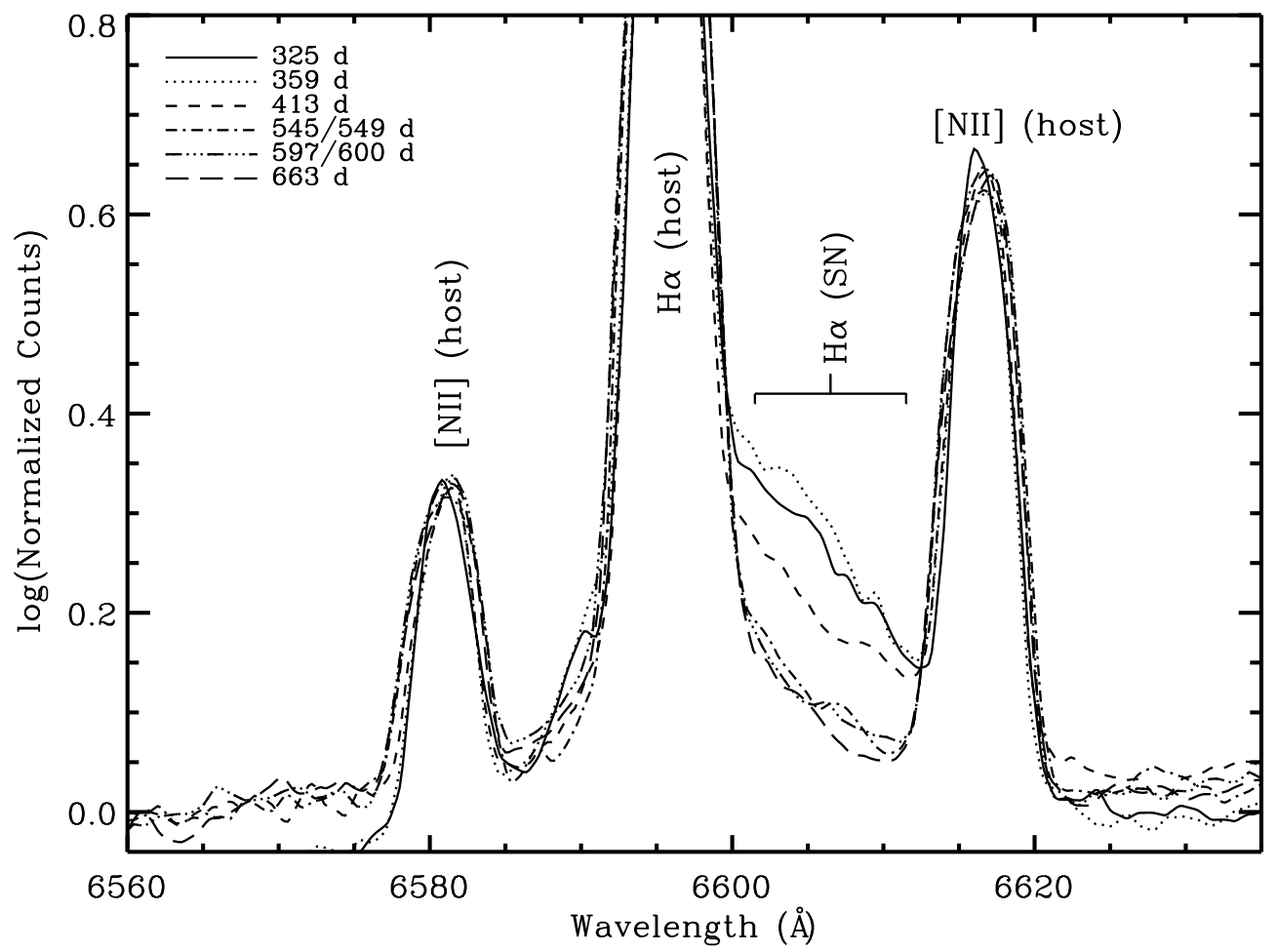

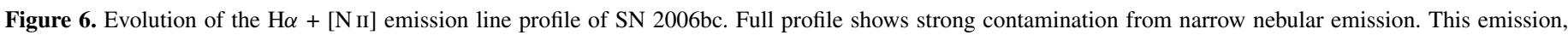
originating in the host galaxy, is labeled as "host." Broad-width emission originating in the SN ejecta is also labeled. 

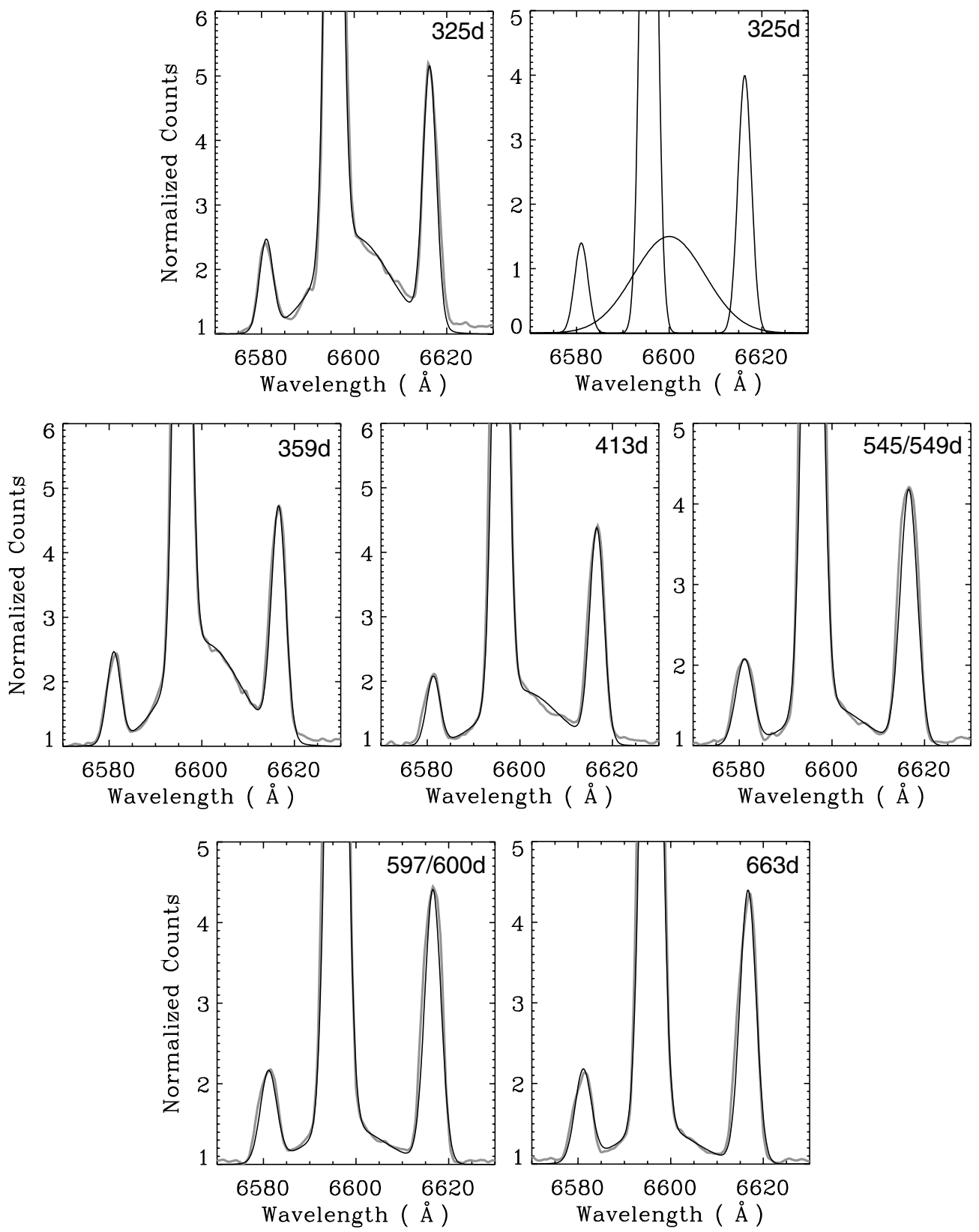

Figure 7. Gaussian fits to the respective $\mathrm{H} \alpha+[\mathrm{N}$ II] profiles observed at each epoch. The fits are shown in black while the data are shown in gray. The fits are comprised of four Gaussian curves, one for each the SN ejecta emission, the galactic [N II] $\lambda 6549$ and $\lambda 6584$ emission, and the galactic $\mathrm{H} \alpha$ emission. As an example, the four constituent parts of the fit for day 325 are given in the top-right panel. The lower panels show the fits for subsequent epochs.

central wavelength evolution of these models which reveals an apparent blueshift of the $\mathrm{H} \alpha$ peak from day 325 to day 663. Figure 8 shows a blueshift of approximately $200 \mathrm{~km} \mathrm{~s}^{-1}$ over the course of our observing program. Although it is a possibility the such a blueshift could be a further consequence of the apparent asymmetrical explosion, blueshifted emission lines are also often the result of the formation of new dust in the ejecta resulting in the emission from the back side of the explosion experiencing greater attenuation than emission from the front side. Consequently, the apparent blueshift of the peak in Figure 8 could be a sign of dust formation in the ejecta of SN $2006 \mathrm{bc}$.

\subsection{Radiative Transfer Modeling}

From our PSF-matched photometry we were successful in obtaining detections in all four IRAC channels on day 537, but we did not detect the SN on day 690 in any channel. Furthermore, we did not obtain detections in the MIPS or IRS/PUI data at any epoch. We combined our IRAC detections on day 537, our $H S T$ results from day 532, and upper limits at $16 \mu \mathrm{m}$ (day 496) and $24 \mu \mathrm{m}$ (day 688) into a single spectral energy distribution (SED) for 2006c approximately 550 days past maximum light. The SED of SN 2006bc is shown in Figure 9. The near- and mid-IR flux measurements are shown in Table 3.

Even with mere upper limits beyond $10 \mu \mathrm{m}$, the IRAC results show evidence for strong IR excesses attributable to warm dust. The dotted line in Figure 9 represents our blackbody fit to the SED. The data are well fitted by the sum of a $4900 \mathrm{~K}$ blackbody, representing the emission from the hot, optically thick ejecta, and a $500 \mathrm{~K}$ modified blackbody due to the emission from a spherical distribution of warm dust with an outer radius of 


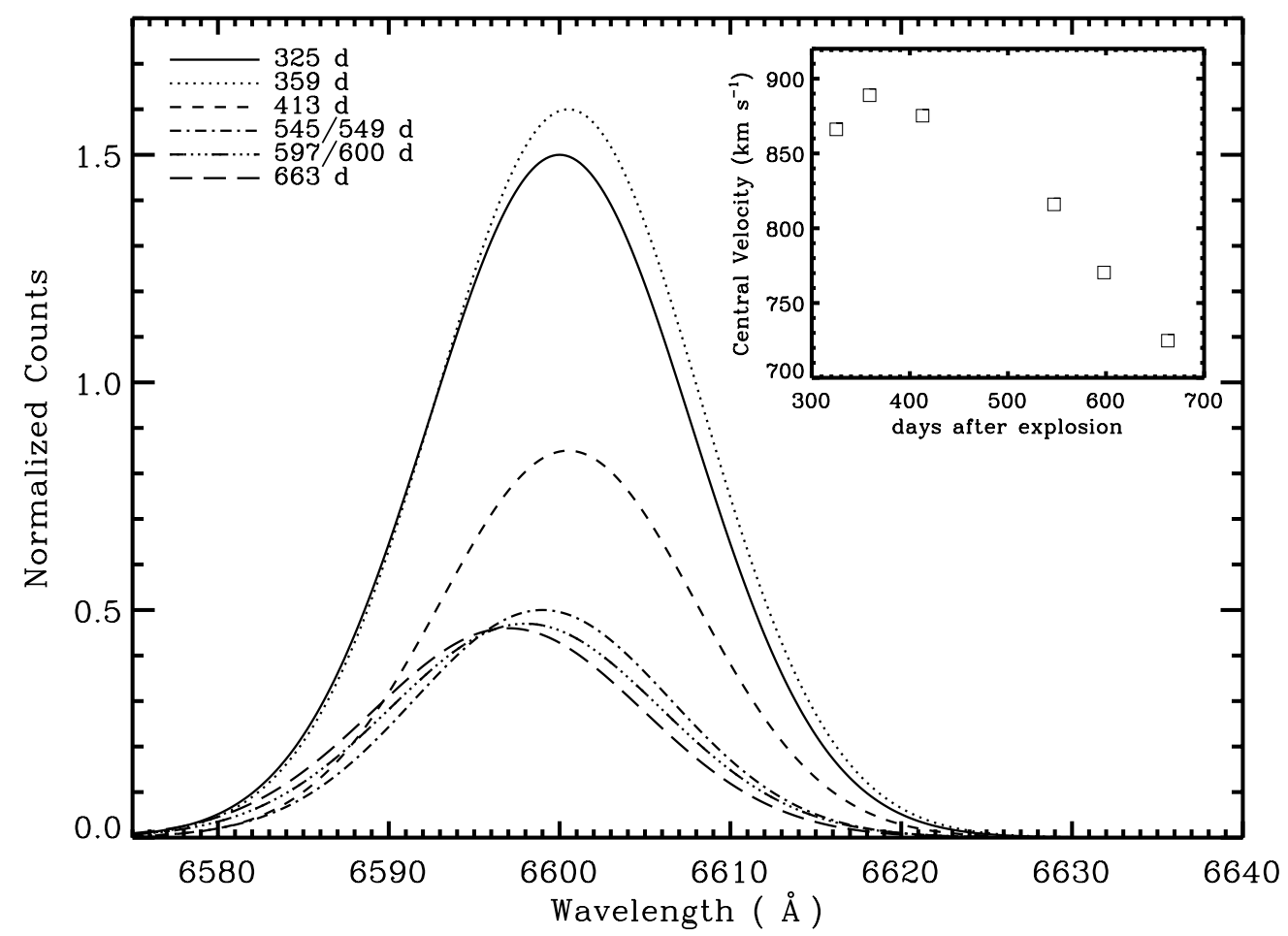

Figure 8. Evolution of the $\mathrm{SN}$ component to our $\mathrm{H} \alpha+[\mathrm{N}$ II] fits from Figure 7. Inset shows the central recessional velocity of the $\mathrm{SN}$ emission vs. time. The plot reveals an apparent blueshift of $\sim 200 \mathrm{~km} \mathrm{~s}^{-1}$ of the SN emission profile from day 325 to 663 .

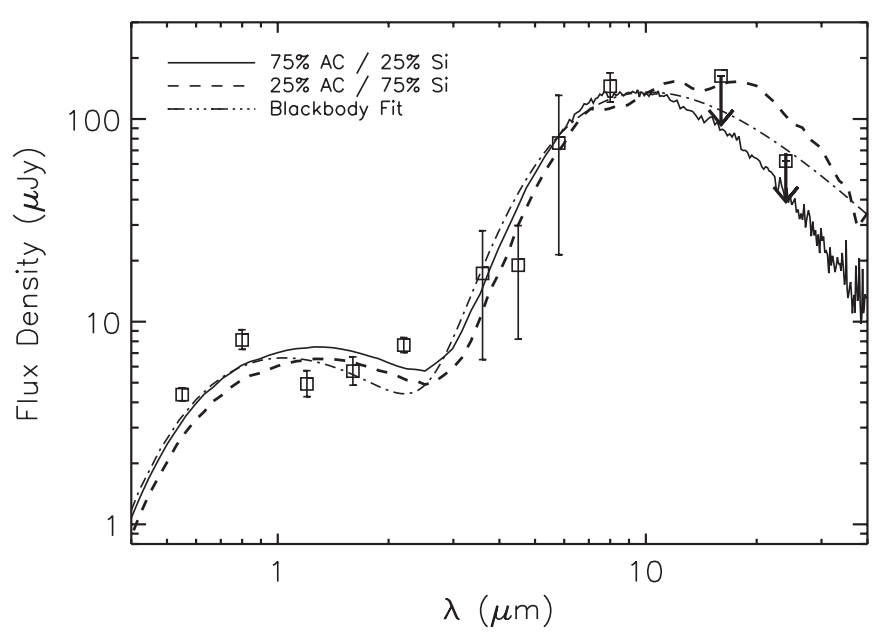

Figure 9. Blackbody and MOCASSIN fits to the SED of SN 2006bc approximately 550 days past maximum light. The optical data were taken on day 532 with $H S T$ /WFPC2. The JHK fluxes come from $H S T$ /NICMOS data taken on day 540. The 3.0, 4.5, 5.8, and $8.0 \mu \mathrm{m}$ fluxes come from Spitzer/IRAC data taken on day 537, while the 16 and $24 \mu \mathrm{m}$ points were obtained with Spitzer using IRS/PUI (day 496) and MIPS (day 688), respectively. The dot-dashed line is the best blackbody fit to the data, the solid line is our best-fit AC-dominated, "smooth" MOCASSIN radiative transfer model, and the dashed line is our best-fit Si-dominated, "smooth" model of the dust around SN 2006bc. The data reveal a strong infrared excess by day 550. Our AC-dominated model represents the better fit to the data (particularly at $24 \mu \mathrm{m}$ ), and predicts $4 \times 10^{-4} M_{\odot}$ of dust around SN 2006bc. If we allow for the dust to form into clumps, the total quantity of dust increases to $2 \times 10^{-3} M_{\odot}$.

$\sim 570$ AU. The modified blackbody is a normal blackbody subject to a $\lambda^{-1}$ emissivity law.

Quantitative modeling of the dust around SN 2006bc proceeded using our three-dimensional Monte Carlo radiative transfer (RT) code MOCASSIN (Ercolano et al. 2005 and references therein). Although we have presented evidence that 2006bc ex-
Table 3

Near- and Mid-IR Fluxes of SN 2006bc for Day 550

\begin{tabular}{lc}
\hline \hline$\lambda$ & $\begin{array}{c}\text { Flux } \\
(\mu \mathrm{m})\end{array}$ \\
\hline 1.2 & $4.9_{-0.8}^{+0.7}$ \\
1.6 & $5.7_{-1.0}^{+0.8}$ \\
2.2 & $7.7_{-0.7}^{+0.6}$ \\
3.6 & $17.3_{-10.8}^{+10.8}$ \\
4.5 & $19.0_{-10.8}^{+10.8}$ \\
5.8 & $76.1_{-57.5}^{+57.5}$ \\
8.0 & $145.0_{-23.9}^{+23.9}$ \\
16.0 & $<163.0$ \\
24.0 & $<62.0$ \\
\hline
\end{tabular}

ploded asymmetrically, we do not have any strong observational constraints on the degree of asymmetry. Consequently, the dust around SN 2006bc was modeled as a spherically expanding shell with an inner radius $R_{\text {in }}$ and an outer radius $R_{\text {out }}$. We modeled the dust around SN 2006bc in two separate ways. We refer to the first one as our "smooth" RT model. Since the source of the luminosity is the ejecta, our smooth model assumes that the source luminosity is spread throughout the shell and proportional to the local density. We further assumed a uniform dust distribution throughout the shell according to an $r^{-2}$ density profile. For our second model, we allow the dust to form into clumps. For this reason, we call it our "clumpy" model. The clumpy model comprises an inhomogeneous distribution of spherical clumps embedded within an interclump medium of a supplied density. The irradiating photons are only produced in this interclump medium with the clumps assumed to be dark. The size of the spherical clumps is given by $r_{c}=0.025\left(r_{\text {out }}\right)$ (Herant \& Woosley 1994; Sugerman et al. 2006) with a volume filling factor $f_{c}=0.20$ and density contrast $\alpha=\rho_{c} / \rho=1 \times 10^{5}$. 
Table 4

Best-fit Monte Carlo Radiative Transfer Models

\begin{tabular}{lccccccc}
\hline \hline Model & $\mathrm{AC} / \mathrm{Si}$ & $\begin{array}{c}T \\
(\mathrm{~K})\end{array}$ & $\begin{array}{c}R_{\text {in }} \\
(\mathrm{AU})\end{array}$ & $\begin{array}{c}R_{\text {out }} \\
(\mathrm{AU})\end{array}$ & $\begin{array}{c}L \\
\left(L_{\odot}\right)\end{array}$ & $\tau_{v}$ & $\begin{array}{c}M_{d} \\
\left(M_{\odot}\right)\end{array}$ \\
\hline Smooth & $0.75 / 0.25$ & 5500 & 67 & 6700 & $1.2 \mathrm{e} 6$ & 1.27 & $4 \mathrm{e}-4$ \\
Clumpy & $0.75 / 0.25$ & 5500 & 67 & 2000 & $1.2 \mathrm{e} 6$ & 1.00 & $2 \mathrm{e}-3$ \\
\hline
\end{tabular}

For a more detailed discussion of modeling a clumpy environment with MOCASSIN, see Ercolano et al. (2007). For each model, we adopted a standard grain size distribution of $a^{-3.5}$ between 0.005 and $0.05 \mu \mathrm{m}$ (Sugerman et al. 2006; Meikle et al. 2007; Kotak et al. 2009). We tested two dust compositions with varying amounts of silicate $(\mathrm{Si})$ and amorphous carbon $(\mathrm{AC})$ dust. The first was the AC-dominated dust (75\% AC, 25\% Si); the second was Si-dominated (25\% AC, $75 \% \mathrm{Si}$ ). Both models used the optical grain constants from Ossenkopf et al. (1992) for Si and Hanner (1988) for AC.

For both the smooth and clumpy models, the primary input parameters that we varied were the ejecta temperature and luminosity, the inner and outer radii of the dust shell, and the mass of dust present. Initial estimates of the ejecta luminosity and the extent of the dust shell came from our optical photometry and the blackbody fits described above, respectively. These parameters, along with the dust mass, were then varied to produce the best fit to the optical/HST and IRAC data points while simultaneously trying to predict more flux than the upper limits at 16 and $24 \mu \mathrm{m}$ would allow.

Our Si- and AC-dominated smooth models are both shown in Figure 9. We found that we were unable to predict sufficiently low flux at $24 \mu \mathrm{m}$ for our silicate-dominated models. This is not unexpected as silicate dust has strong emission features at 10 and $18 \mu \mathrm{m}$ which acts to hold up the SED flux relative to that at 3-8 $\mu \mathrm{m}$. Our best-fit model, therefore, corresponds to dust with a composition of $75 \% \mathrm{AC}$ and $25 \%$ silicate dust. The other best-fit parameters are an ejecta temperature of $5500 \mathrm{~K}$, an ejecta luminosity of $1.2 \times 10^{6} L_{\odot}$, a dust shell inner radius and outer radius of $67 \mathrm{AU}$ and $6700 \mathrm{AU}$, respectively, and a total dust mass of $4 \times 10^{-4} M_{\odot}$ (see Table 4). This corresponds to an $A_{v}=1.4$. If we allow the dust to form in clumps, the total amount of dust becomes $\sim 2.0 \times 10^{-3} M_{\odot}$. Even if all of this dust formed during the $\mathrm{SN}$ explosion, it would still be orders of magnitude less than what a typical core-collapse SN would need to form in order to explain the amount of dust observed in the early universe (Bertoldi et al. 2003; Morgan \& Edmunds 2003).

\subsection{Possible Light Echo}

The optical and near-IR light curves of SN 2006bc are presented in Figure 10. After difference imaging of our GMOS data, detections were made and magnitudes were measured on days 325,359 , and 413 . The data on days 532 and 694 are from HST/WFPC2. The photometry has not been corrected for foreground extinction. SN 2006bc undergoes a steep drop immediately following maximum light leading us to categorize $2006 \mathrm{bc}$ as a Type II-L SN. Assuming a total foreground extinction of $A_{v} \sim 1.6$ (see above) and a distance to NGC 2397 of $20.5 \mathrm{Mpc}$, SN 2006bc reached a maximum brightness of $V \sim-17.2$. Although showing normal peak brightness for a core-collapse $\mathrm{SN}, 2006 \mathrm{bc}$ then fades by $\sim 6$ mag during the first 400 days, suggesting a relatively low ${ }^{56} \mathrm{Ni}$ yield in the SN explosion (Kasen \& Woosley 2009) and/or an increase in extinction due to new dust. Typically, such steep declines are seen in underluminous $\mathrm{SNe}$, though two Type II plateau

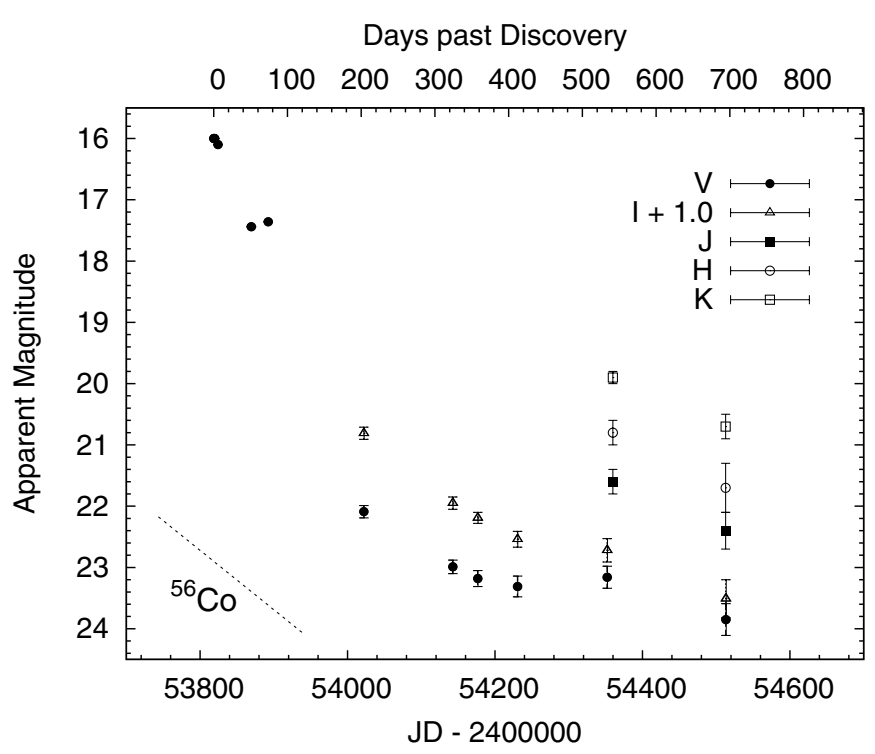

Figure 10. $V I_{c} J H K$ light curve of SN 2006bc. The data within the first 10 days are unfiltered discovery magnitudes while the data between days 40 and 60 comes from Astrosurf.com. The $V$ and $I$ data points from day 203 are measured from the ACS WFC images taken by Smartt et al. (2009). The data beyond 300 days come from the observations made for our study. The light curves show an abrupt drop of 7-8 mag during the first 400 days past maximum light. This is likely due to a combination of a low ${ }^{56} \mathrm{Ni}$ yield in the explosion and an increased optical extinction due to dust formation. The data also show a short-lived flattening in the light curve from $\sim 400$ to 500 days past maximum due to an optical light echo from CSM.

SNe, 1994W (Sollerman et al. 1998) and 2007od (Andrews et al. 2010), were very highly luminous $(V<-18)$ during the photospheric stage and yet showed low late-time luminosities attributed to a combination of low ${ }^{56} \mathrm{Ni}$ mass and dust extinction.

By the onset of the nebular phase, SN 2006bc begins to decline with a rate in $V$ and $I_{c}$ that is consistent with the radioactive decay rate ${ }^{56} \mathrm{Co}$. However, we observe an approximate 100 day flattening of the light curve in both $V$ and $I_{c}$ from days 416 to 541 , after which a ${ }^{56} \mathrm{Co}$ decline rate resumes. The most likely explanation for this phenomenon is an optical light echo, and given the narrow time window in which the flattening was observed, we can place constraints on both the distance and geometry of the CSM responsible for the echo. There are two obvious geometries that could explain the flattening of the light curve. The first one has the CSM lying in a torus surrounding the SN. In this case, due to light travel effects, the late onset and short duration of the flattening require that the torus to be oriented close to face-on and have a thickness of no more than $\sim 100$ lt-days. Furthermore, the fact that flattening begins around day 413 suggests that the inner edge of the torus or ring must lie approximately $400 \mathrm{lt}$-days $(1.04 \mathrm{e} 18 \mathrm{~cm})$ from the geometric center of the explosion. This is consistent with the size of the main ring around 1987A.

Another possible scenario is that the CSM is located within a thin spheroidal shell with a radius of approximately 400-500 lt-days. Given the time it would take for light to travel to all parts of the shell and then be scattered in the direction to Earth, one is likely to assume that such a geometry could not produce the short-duration flattening that we have observed. However, Sugerman et al. (2005) have shown the following proportion to be an empirically close fit to the average scattering function for $\mathrm{MRN}^{9}$ dust over scattering angles between $\theta=0^{\circ}$ to $\sim 160^{\circ}$.

\footnotetext{
9 Mathis et al. (1977)
} 
The echo brightness scales as

$$
\text { E.B. } \propto\left[1.0+2.21 \times 10^{-3} \times(\theta)^{2}\right]^{-1.123} .
$$

This relation indicated that the echo brightness falls off sharply with increasing scattering angle, $\theta$. With a thin shell of radius 400-500 lt-days, the scattered light from the CSM at low $\theta$ will begin to flatten out the light curve 400-500 days after the explosion while the material at increasing $\theta$ could continue to hold the light curve up for the subsequent 100 days. Either of these scenarios are possible, though the latter seems more likely as the former requires a very specific inclination $(i \gtrsim 80)$ of the torus to fit the data.

\section{CONCLUSIONS}

We have presented multi-temporal, multi-wavelength observations of the core-collapse SN 2006bc during its nebular phase. Based on an analysis of these observations we have drawn the following conclusions.

1. An analysis of the evolution of the optical spectra of SN 2006bc shows evidence that the average central wavelength of the $\mathrm{SN} \mathrm{H} \alpha$ emission profile is redshifted relative to the nebular emission from the galaxy between 325 and 663 days. This is likely due to the asymmetric nature of the explosion. The fact that this same redshift was not seen in the emission lines of [O I] suggests the asymmetry was of the iron-peak elements only. Furthermore, we find evidence for a gradual blueshifting of the $\mathrm{H} \alpha$ emission line profile during our observing period due to the formation of new dust within the SN ejecta.

2. RT modeling of the dust around $2006 \mathrm{bc}$ has revealed an upper limit of $4 \times 10^{-4} M_{\odot}$ of new dust having formed during the $\mathrm{SN}$ explosion. If we allow the dust to form into clumps, our models predict as much as $2 \times 10^{-3} M_{\odot}$ of new dust formed during the explosion of SN $2006 \mathrm{bc}$. These results are consistent with the mounting evidence suggesting dust formation within the ejecta of core-collapse $\mathrm{SNe}$ is insufficient to explain the large quantities of dust observed in the early universe.

3. An approximate 100 day flattening of the light curve beginning $\sim 400$ days past explosion provides evidence for a light echo off a thin, spheroidal shell of CSM surrounding SN 2006bc at a distance of 400-500 lt-day.

This work has been supported by NSF grant AST-0707691 and HST grant HST-GO-11229.03-A. This work is based in part on observations made with the Spitzer Space Telescope, which is operated by the Jet Propulsion Laboratory, California Institute of Technology, under a contract with NASA. A portion of this data was obtained at the Gemini Observatory, which is operated by the Association of Universities for Research in Astronomy (AURA) under a cooperative agreement with the NSF on behalf of the Gemini partnership. The standard data acquisition has been supported by NSF grants AST-0503871 and AST-0803158 to A. U. Landolt.

\section{Facilities: Gemini:South, HST, Spitzer}

\section{REFERENCES}

Andrews, J. E., Gallagher, J. S., Clayton, G. C., et al. 2010, ApJ, 715, 541 Andrews, J. E., Sugerman, B. E. K., Clayton, G. C., et al. 2011, ApJ, 731, 47
Barbon, R., Benetti, S., Rosino, L., Cappellaro, E., \& Turatto, M. 1990, A\&A, 237, 79

Bertoldi, F., Cox, P., Neri, R., et al. 2003, A\&A, 409, L47

Cernuschi, F., Marsicano, F., \& Codina, S. 1967, Ann. Astrophys., 30, 1039

Chugai, N. N. 1991, SvA, 35, 171

Chugai, N. N., Fabrika, S. N., Sholukhova, O. N., et al. 2005, Astron. Lett., 31, 792

Deneault, E., Clayton, D. D., \& Heger, A. 2003, ApJ, 594, 312

Dolphin, A. E. 2000, PASP, 112, 1397

Dolphin, A. E. 2009, PASP, 121, 655

Draine, B. T. 2009, in ASP Conf. Ser. 414, Cosmic Dust-Near and Far, ed. T. Henning, E. Grün, \& J. Steinacker (San Francisco, CA: ASP), 453

Dwek, E., \& Cherchneff, I. 2011, ApJ, 727, 63

Dwek, E., Galliano, F., \& Jones, A. P. 2007, ApJ, 662, 927

Elmhamdi, A., Danziger, I. J., Cappellaro, E., et al. 2004, A\&A, 426, 963

Elmhamdi, A., Danziger, I. J., Chugai, N., et al. 2003, MNRAS, 338, 939

Ercolano, B., Barlow, M. J., \& Storey, P. J. 2005, MNRAS, 362, 1038

Ercolano, B., Barlow, M. J., \& Sugerman, B. E. K. 2007, MNRAS, 375, 753

Fabbri, J., Otsuka, M., Barlow, M. J., et al. 2011, MNRAS, 418, 1285

Fox, O., Skrutskie, M. F., Chevalier, R. A., et al. 2009, ApJ, 691, 650

Gehrz, R. 1989, in IAU Symp. 135, Interstellar Dust, ed. L. J. Allamandola \& A. G. G. M. Tielens (Dordrecht: Kluwer), 445

Hanner, M. 1988, in Infrared Observations of Comets Halley and Wilson and Properties of the Grains, ed. M. S. Hanner (Washington, DC: NASA Sci. Tech. Info. Div.), 22

Hendry, M. A., Smartt, S. J., Maund, J. R., et al. 2005, MNRAS, 359, 906

Herant, M., \& Woosley, S. E. 1994, ApJ, 425, 814

Immler, S., \& Brown, P. J. 2006, ATel, 776, 1

Kasen, D., \& Woosley, S. E. 2009, ApJ, 703, 2205

Kotak, R., Meikle, P., Pozzo, M., et al. 2006, ApJ, 651, L117

Kotak, R., Meikle, W. P. S., Farrah, D., et al. 2009, ApJ, 704, 306

Krist, J., \& Hook, R. 2004, The Tiny Tim User's Manual (Baltimore, MD: STScI)

Landolt, A. U. 2009, AJ, 137, 4186

Lucy, L. B., Danziger, I. J., Gouiffes, C., \& Bouchet, P. 1991, in Supernovae, ed. S. E. Woosley (New York: Springer), 82

Maiolino, R., Nagao, T., Marconi, A., et al. 2006, Mem. Soc. Astron. Ital., 77, 643

Makovoz, D., \& Khan, I. 2005, in ASP Conf. Ser. 347, Astronomical Data Analysis Software and Systems XIV, ed. P. Shopbell, M. Britton, \& R. Ebert (San Farncisco, CA: ASP), 81

Martin, R. 2006, CBET, 446, 1

Mathis, J. S., Rumpl, W., \& Nordsieck, K. H. 1977, ApJ, 217, 425

Mattila, S., Meikle, W. P. S., Lundqvist, P., et al. 2008, MNRAS, 389, 141

Meikle, W. P. S., Mattila, S., Pastorello, A., et al. 2007, ApJ, 665, 608

Meikle, W. P. S., Spyromilio, J., Allen, D. A., Varani, G., \& Cumming, R. J. 1993, MNRAS, 261, 535

Meikle, W. P. S., Spyromilio, J., Varani, G., \& Allen, D. A. 1989, MNRAS, 238, 193

Michałowski, M. J., Murphy, E. J., Hjorth, J., et al. 2010, A\&A, 522, A15

Morgan, H. L., \& Edmunds, M. G. 2003, MNRAS, 343, 427

Nozawa, T., Kozasa, T., Umeda, H., Maeda, K., \& Nomoto, K. 2003, ApJ, 598, 785

Ossenkopf, V., Henning, T., \& Mathis, J. S. 1992, A\&A, 261, 567

Patat, F., Baade, D., \& Wang, L. 2006, CBET, 450, 1

Phillips, M. M., \& Williams, R. E. 1991, in Supernovae, ed. S. E. Woosley (New York: Springer-Verlag), 36

Poznanski, D., Gal-Yam, A., Maoz, D., et al. 2002, PASP, 114, 833

Pozzo, M., Meikle, W. P. S., Fassia, A., et al. 2004, MNRAS, 352, 457

Sahu, D. K., Anupama, G. C., Srividya, S., \& Muneer, S. 2006, MNRAS, 372, 1315

Smartt, S. J., Eldridge, J. J., Crockett, R. M., \& Maund, J. R. 2009, MNRAS, 395, 1409

Smith, N., Foley, R. J., \& Filippenko, A. V. 2008, ApJ, 680, 568

Smith, N., Silverman, J. M., Chornock, R., et al. 2009, ApJ, 695, 1334

Sollerman, J., Cumming, R. J., \& Lundqvist, P. 1998, ApJ, 493, 933

Sugerman, B. E. K., Crotts, A. P. S., Kunkel, W. E., Heathcote, S. R., \& Lawrence, S. S. 2005, ApJS, 159, 60

Sugerman, B. E. K., Ercolano, B., Barlow, M. J., et al. 2006, Science, 313, 196 Todini, P., \& Ferrara, A. 2001, MNRAS, 325, 726

Tomaney, A. B., \& Crotts, A. P. S. 1996, AJ, 112, 2872

Tully, R. B., Rizzi, L., Shaya, E. J., et al. 2009, AJ, 138, 323

Valiante, R., Schneider, R., Bianchi, S., \& Andersen, A. C. 2009, MNRAS, 397, 1661

Welch, D. L., Clayton, G. C., Campbell, A., et al. 2007, ApJ, 669, 525 